Wei Lian and Runzhang $\mathrm{Xu}$ *

\title{
Global well-posedness of nonlinear wave equation with weak and strong damping terms and logarithmic source term
}

https://doi.org/10.1515/anona-2020-0016

Received December 9, 2018; accepted January 18, 2019.

Abstract: The main goal of this work is to investigate the initial boundary value problem of nonlinear wave equation with weak and strong damping terms and logarithmic term at three different initial energy levels, i.e., subcritical energy $E(0)<d$, critical initial energy $E(0)=d$ and the arbitrary high initial energy $E(0)>$ $0(\omega=0)$. Firstly, we prove the local existence of weak solution by using contraction mapping principle. And in the framework of potential well, we show the global existence, energy decay and, unlike the power type nonlinearity, infinite time blow up of the solution with sub-critical initial energy. Then we parallelly extend all the conclusions for the subcritical case to the critical case by scaling technique. Besides, a high energy infinite time blow up result is established.

Keywords: Wave equation; global solution; weak and strong damping terms; energy decay; infinite time blow up; logarithmic nonlinearity

\section{Introduction and main results}

In this paper, we study initial boundary value problem of nonlinear wave equation with weak and strong damping terms and logarithmic source term

$$
\begin{aligned}
& u_{t t}-\Delta u-\omega \Delta u_{t}+\mu u_{t}=u \ln |u|,(x, t) \in \Omega \times(0, \infty), \\
& u(x, t)=0, x \in \partial \Omega, t \geq 0, \\
& u(x, 0)=u_{0}(x), u_{t}(x, 0)=u_{1}(x), x \in \Omega,
\end{aligned}
$$

where $\Omega \subset \mathbb{R}^{n}(n \geq 1)$ is a bounded domain with a smooth boundary $\partial \Omega$,

$$
\omega \geq 0, \quad \mu>-\omega \lambda_{1},
$$

$\lambda_{1}$ being the first eigenvalue of the operator $-\Delta$ under homogeneous Dirichlet boundary conditions.

The undamped hyperbolic equation

$$
u_{t t}-\Delta u=f(u),
$$

was introduced by D'Alembert [1] to model the propagation of waves along vibrating elastic string. By introducing the potential well, the global existence and finite time blow up of solution to (1.5) with $E(0)<d$ were proved by Payne and Sattinger in [2], [3] respectively.

The nonlinear wave equation with linear weak damping term was considered by Levine and Serrin [4] in abstract form

$$
P u_{t t}+A(u)+Q\left(t, u_{t}\right)=F(u)
$$

\footnotetext{
Wei Lian, College of Automation, Harbin Engineering University, People's Republic of China

*Corresponding Author: Runzhang Xu, College of Automation, Harbin Engineering University, People's Republic of China and College of Mathematical Sciences, Harbin Engineering University, People's Republic of China
} 
and they proved the nonexistence of global solution for the negative initial energy, i.e., $E(0)<0$. Later, Pucci and Serrin [5] extended this results to $E(0)<D_{1}$, where $D_{1}$ is positive. Vitillaro [6] studied the similar problem replacing the linear weak damping term by the nonlinear one

$$
u_{t t}-\Delta u+b\left|u_{t}\right|^{m-2} u_{t}=c|u|^{p-2} u
$$

and derived the conditions of initial data leading to finite time blow up of the solution for $E(0) \leq E_{1}$.

For the initial boundary value problem of classical strongly damped wave equation

$$
u_{t t}-\Delta u-\Delta u_{t}=f(u),
$$

Webb [7] gave the local existence uniqueness, global existence and the asymptotic behavior of the solution. For the initial boundary value problem of strongly damped semilinear wave equation

$$
u_{t t}-\omega \Delta u_{t}-\Delta u+\phi(u)=f,
$$

Pata and Squassina [8] proved the existence of the universal attractor, in the presence of a quite general nonlinearity of critical growth. Moreover, they obtained the asymptotic behavior of the solutions in dependence of the damping coefficient.

For the wave equation with both linear weak and strong damping terms, we can directly go to [9] for the most recent progress. Gazzola and Squassina [9] proved that the initial boundary value problem of the weak and strong damping hyperbolic equation

$$
u_{t t}-\Delta u+\mu u_{t}-\omega \Delta u_{t}=|u|^{p-2} u,
$$

has a unique local solution, and the global existence and nonexistence results were also proved for $E(0) \leq d$. Also, the finite time blow up of solution with high energy $E(0)>d(\omega=0, \mu>0)$ was obtained.

The logarithmic nonlinearity is of much interest in physics, since it appears naturally in inflation cosmology and super symmetric filed theories, quantum mechanics and nuclear physics [10], [11]. Haraux and Cazenave[12] proved the existence and uniqueness of solution for the Cauchy problem for the nonlinear Schrödinger equation

$$
i u_{t}+\Delta u+V u+k u \log |u|^{2}=0 \text { in } \mathbb{R}^{N} \times \mathbb{R}_{+}
$$

and for the nonlinear Klein-Gordon equation

$$
u_{t t}-\Delta u=k u \log |u|^{2} \text { in } \mathbb{R}^{3} \times \mathbb{R}_{+} .
$$

Górka [13] obtained that the initial boundary value problem of logarithmic Klein-Gordon equation

$$
\begin{cases}u_{t t}-u_{x x}+u=\varepsilon u \log |u|^{2}, & x \in \mathcal{O}, t \in[0, T), \\ u(x, 0)=u_{0}(x), \quad u_{t}(x, 0)=u_{1}(x), & x \in \mathcal{O}, \\ u(x, t)=0, & x \in \partial \mathcal{O}, t \in[0, T),\end{cases}
$$

where $\mathcal{O}$ is a finite interval $V=[a, b]$, admits global weak solutions for all $\left(u_{0}, u_{1}\right) \in H_{0}^{1} \times L^{2}$ and $\varepsilon \in[0,1]$ in one-dimensional case.

The weak damping with logarithmic wave equation

$$
u_{t t}-\Delta u+u_{t}+u+|u|^{2} u=u \ln |u|^{2}
$$

was introduced by Hiramatsu [14] to model the dynamics of Q-ball in theoretical physics. Then its initial boundary value problem was considered by Han in [15]

$$
\left\{\begin{array}{l}
u_{t t}-\Delta u+u+u_{t}+|u|^{2} u=u \ln |u|^{2}, \quad x \in \Omega, t \in[0, T), \\
u(x, 0)=u_{0}(x), \quad u_{t}(x, 0)=u_{1}(x), \quad x \in \Omega, \\
u(x, t)=0, \quad x \in \partial \Omega, t \in[0, T)
\end{array}\right.
$$


and the global existence of weak solutions was proved, for all $\left(u_{0}, u_{1}\right) \in H_{0}^{1} \times L^{2}$ in $\mathbb{R}^{3}$. By constructing an appropriate Lyapunov function, Zhang and Liu [16] obtained the exponential decay estimates of energy with $E(0)<d$ for all $\left(u_{0}, u_{1}\right) \in H_{0}^{1} \times L^{2}, I\left(u_{0}\right)>0$.

Al-Gharabli and Messaoudi [17] proved the global existence and the exponential decay of solutions of the following plate equation

$$
\begin{cases}u_{t t}+\Delta^{2} u+u+u_{t}=k u \ln |u|, & x \in \Omega, t>0, \\ u(x, t)=\frac{\partial u}{\partial v}(x, t)=0, & x \in \partial \Omega, t \geq 0, \\ u(x, 0)=u_{0}(x), u_{t}(x, 0)=u_{1}(x), & x \in \Omega,\end{cases}
$$

for all $\left(u_{0}, u_{1}\right) \in H_{0}^{2}(\Omega) \times L^{2}(\Omega)$ and $0<E(0)<d, I\left(u_{0}\right)>0$. Later, Al-Gharabli and Messaoudi [18] considered a general damping $h\left(u_{t}\right)$ instead of a linear $u_{t}$ one considered in [17], where $h$ is a function having a polynomial growth near the origin. They established the global existence and the general decay of solutions for all $\left(u_{0}, u_{1}\right) \in H_{0}^{2}(\Omega) \times L^{2}(\Omega), 0<E(0)<d$ and $I\left(u_{0}\right)>0$.

As shown in the previous works, the dynamical behaviors of solutions are quite different when with presence of different nonlinearities, i.e., power type and logarithmic type. To be specific, with the presence of logarithmic term, the finite time blow up of solution don't occur anymore which means the Nehari manifold in the initial energy space $H_{0}^{1}$ no longer plays a role as a threshold separating global and non-global existence of solution. And instead in this article the results show that Nehari manifold can be viewed as a threshold which indicates the decay or infinite time blow up of solutions. In order to investigate and describe the dynamical behavior of solution that strongly relied on the initial data, we focus on the logarithmic term in three initial data levels, i.e., subcritical energy $E(0)<d$, critical initial energy $E(0)=d$ and the arbitrary high initial energy $E(0)>0(\omega=0)$.

The present paper is organized as follows. Section 2 presents some notations and preliminaries. In section 3, we state our main results. Section 4 prove the local existence of solution. Section 5 prove the global existence, asymptotic behavior and infinite time blow up of solution for $E(0)<d$. In section 6 , the global existence, asymptotic behavior and blowup of solution for $E(0)=d$ is proved. At last, in Section 7, we prove the infinite time blowup result for $E(0)>0(\omega=0)$.

\section{Notations and primary lemmas}

In this section, we present some preliminaries to prove the main results. We denote the inner product and the norm on $H_{0}^{1}(\Omega)$ by $(\cdot, \cdot)$ and $\|\nabla \cdot\|$, respectively. The symbol $\|\cdot\|$ will indicate the norm on $L^{2}(\Omega)$. Moreover, we denote by $\langle\cdot, \cdot\rangle\rangle$ the duality pairing between $H^{-1}(\Omega)$ and $H_{0}^{1}(\Omega)$. For any $v, w \in H_{0}^{1}(\Omega)$, we have

$$
(v, w)_{\star}=\omega \int_{\Omega} \nabla v \nabla w+\mu \int_{\Omega} v w
$$

and

$$
\|v\|_{\star}=(v, v)_{\star}^{\frac{1}{2}} .
$$

By (1.4), $\|\cdot\| \star$ is an equivalent eccentric module over $H_{0}^{1}(\Omega)$.

First, for problem (1.1)-(1.3) we introduce the energy functional

$$
E(t)=\frac{1}{2}\left\|u_{t}\right\|^{2}+\frac{1}{2}\|\nabla u\|^{2}-\frac{1}{2} \int_{\Omega} u^{2} \ln |u| d x+\frac{1}{4}\|u\|^{2},
$$

the potential energy functional

$$
J(u)=\frac{1}{2}\|\nabla u\|^{2}-\frac{1}{2} \int_{\Omega} u^{2} \ln |u| d x+\frac{1}{4}\|u\|^{2}
$$


and the Nehari functional

$$
I(u)=\|\nabla u\|^{2}-\int_{\Omega} u^{2} \ln |u| d x .
$$

By a direct computation,

$$
J(u)=\frac{1}{2} I(u)+\frac{1}{4}\|u\|^{2} .
$$

By $I(u)$ we define the potential well (stable set)

$$
W=\left\{u \in H_{0}^{1}(\Omega) \mid I(u)>0\right\} \cup\{0\},
$$

the outer space of potential well (unstable set)

$$
V=\left\{u \in H_{0}^{1}(\Omega) \mid I(u)<0\right\}
$$

and the Nehari manifold

$$
\mathcal{N}=\left\{u \in H_{0}^{1}(\Omega) \backslash\{0\} \mid I(u)=0\right\} .
$$

The depth of potential well is defined as

$$
d=\inf _{u \in \mathcal{N}} J(u) .
$$

On the other hand, as the difference between two types of nonlinearities, assumptions on the power type nonlinearity don't work on the logarithmic one. Consequently, introducing the logarithmic Sobolev inequality and revisiting the corresponding estimates is a necessity to handle logarithmic nonlinear term $u \ln |u|$. The following logarithmic Sobolev inequality was introduced by [20], Chapter 8.14 (also see [19] for a different proof).

Lemma 2.1. $[19,20]$ If $u \in H^{1}\left(\mathbb{R}^{n}\right)$ and $a>0$. Then

$$
2 \int_{\mathbb{R}^{n}}|u(x)|^{2} \ln \left(\frac{|u(x)|}{\|u\|}\right) d x+n(1+\ln a)\|u\|^{2} \leq \frac{a^{2}}{\pi} \int_{\mathbb{R}^{n}}|\nabla u(x)|^{2} d x .
$$

For $u \in H_{0}^{1}(\Omega)$, we can define $u(x)=0$ for $x \in \mathbb{R}^{n} \backslash \Omega$. Then $u \in H^{1}\left(\mathbb{R}^{n}\right)$, that is to say, for a general domain $\Omega$, we have following logarithmic Sobolev inequality,

$$
2 \int_{\Omega}|u(x)|^{2} \ln \left(\frac{|u(x)|}{\|u\|}\right) d x+n(1+\ln a)\|u\|^{2} \leq \frac{a^{2}}{\pi} \int_{\Omega}|\nabla u(x)|^{2} d x,
$$

where $u \in H_{0}^{1}(\Omega)$ and $a>0$.

Lemma 2.2. Assume that $u \in H_{0}^{1}(\Omega) \backslash\{0\}$. Then we have

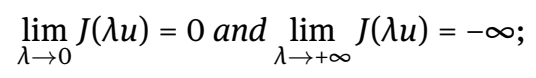

(ii) There exists a unique $\lambda^{\star}=\lambda^{\star}(u)$ such that $\left.\frac{d}{d \lambda} J(\lambda u)\right|_{\lambda=\lambda^{*}}=0$, where $\lambda^{*}=\exp \left(\frac{\|\nabla u\|^{2}-\int_{\Omega} u^{2} \ln |u| d x}{\|u\|^{2}}\right)$;

(iii) $J(\lambda u)$ is increasing on the interval $\left(0, \lambda^{\star}\right)$, decreasing on the interval $\left(\lambda^{\star},+\infty\right)$ and attains its maximum unique $\lambda^{\star} \in(0,+\infty)$ such that

$$
I(\lambda u)\left\{\begin{array}{l}
>0,0<\lambda<\lambda^{*} \\
=0, \lambda=\lambda^{*} \\
<0, \lambda^{*}<\lambda<+\infty
\end{array}\right.
$$


Proof. (i) We get

$$
\begin{aligned}
J(\lambda u) & =\frac{\lambda^{2}}{2}\|\nabla u\|^{2}-\frac{\lambda^{2}}{2} \int_{\Omega} u^{2} \ln |\lambda u| d x+\frac{\lambda^{2}}{4}\|u\|^{2} \\
& =\frac{\lambda^{2}}{2}\|\nabla u\|^{2}-\frac{\lambda^{2}}{2} \int_{\Omega} u^{2} \ln |u| d x-\frac{\lambda^{2}}{2} \ln |\lambda|\|u\|^{2}+\frac{\lambda^{2}}{4}\|u\|^{2} \\
& =\frac{\lambda^{2}}{2}\left(\|\nabla u\|^{2}-\int_{\Omega} u^{2} \ln |u| d x-\ln |\lambda|\|u\|^{2}+\frac{1}{2}\|u\|^{2}\right),
\end{aligned}
$$

which tells $\lim _{\lambda \rightarrow 0} J(\lambda u)=0$ and $\lim _{\lambda \rightarrow+\infty} J(\lambda u)=-\infty$.

(ii) A direct computation on (2.10) yields

$$
\begin{aligned}
\frac{d}{d \lambda} J(\lambda u) & =\lambda\|\nabla u\|^{2}-\lambda \int_{\Omega} u^{2} \ln |u| d x-\lambda \ln |\lambda|\|u\|^{2} \\
& =\lambda\left(\|\nabla u\|^{2}-\int_{\Omega} u^{2} \ln |\lambda u| d x\right),
\end{aligned}
$$

(iii) The conclusion (iii) directly follows from

$$
\begin{aligned}
I(\lambda u) & =\lambda^{2}\|\nabla u\|^{2}-\lambda^{2} \int_{\Omega} u^{2} \ln |u| d x-\lambda^{2} \ln |\lambda|\|u\|^{2} \\
& =\lambda \frac{d J(\lambda u)}{d \lambda}
\end{aligned}
$$

and conclusion (ii).

Lemma 2.3. Let $u \in H_{0}^{1}(\Omega)$ and $r:=(2 \pi)^{\frac{n}{4}} e^{\frac{n}{2}}$.

(i) If $0<\|u\| \leq r$, then $I(u) \geq 0$;

(ii) If I $(u)<0$, then $\|u\|>r$;

(iii) If I $(u)=0$ and $\|u\|=0$, then $\|u\| \geq r$.

Proof. (i) Using the logarithmic Sobolev inequality (2.9), for $a>0$, we get

$$
\begin{aligned}
I(u) & =\|\nabla u\|^{2}-\int_{\Omega} u^{2} \ln |u| d x \\
& =\|\nabla u\|^{2}-\int_{\Omega} u^{2}\left(\ln \frac{|u|}{\|u\|}+\ln \|u\|\right) d x \\
& \geq\left(1-\frac{a^{2}}{2 \pi}\right)\|\nabla u\|^{2}+\frac{n(1+\ln a)}{2}\|u\|^{2}-\|u\|^{2} \ln \|u\| .
\end{aligned}
$$

Taking $a=\sqrt{2 \pi}$, we gain

$$
I(u) \geq\left(\frac{n(2+\ln (2 \pi))}{4}-\ln \|u\|\right)\|u\|^{2} .
$$

If $0<\|u\| \leq r$, then $\frac{n(2+\ln (2 \pi))}{4} \geq \ln \|u\|$, which gives $I(u) \geq 0$. 
(ii) From (2.13) and $I(u)<0$, we can see that

$$
\left(\frac{n(2+\ln (2 \pi))}{4}-\ln \|u\|\right)\|u\|^{2}<0
$$

which means

$$
\|u\|>(2 \pi)^{\frac{n}{4}} e^{\frac{n}{2}}=r .
$$

(I) This conclusion is similar to the proof of (ii).

Lemma 2.4. The depth of potential well $d$ in (2.8) satisfies

$$
d \geq \frac{1}{4}(2 \pi)^{\frac{n}{2}} e^{n}
$$

Proof. From the definition of $d$ in (2.8) and Lemma 2.3 in (iii), it follows that $u \in \mathcal{N}$. As a result, we obtain

$$
0=I(u) \geq\left(\frac{n(2+\ln (2 \pi))}{4}-\ln \|u\|\right)\|u\|^{2},
$$

which implies

$$
\|u\| \geq(2 \pi)^{\frac{n}{4}} e^{\frac{n}{2}}
$$

By virtue of (2.4), $I(u)=0$ and (2.16), we obtain

$$
\begin{aligned}
J(u) & =\frac{1}{2} I(u)+\frac{1}{4}\|u\|^{2} \\
& =\frac{1}{4}\|u\|^{2} \\
& \geq \frac{1}{4}(2 \pi)^{\frac{n}{2}} e^{n},
\end{aligned}
$$

which gives (2.14).

Lemma 2.5. Let $u$ be a solution of problem (1.1)-(1.3), then $E(t)$ is a non-increasing function with respect to $t$.

Proof. Multiplying Eq. (1.1) by $u_{t}$ and integrating it over $\Omega \times[s, t)$, we can obtain

$$
E(t)+\int_{s}^{t}\left\|u_{t}(\tau)\right\|_{\star}^{2} d \tau=E(s) .
$$

Thus, the proof is completed.

Definition 2.6. Function $u=u(x, t)$ is called a weak solution of problem (1.1)-(1.3) over $\Omega \times[0, T]$, if

$$
u \in C\left([0, T], H_{0}^{1}(\Omega)\right) \cap C^{1}\left([0, T], L^{2}(\Omega)\right) \cap C^{2}\left([0, T], H^{-1}(\Omega)\right),
$$

$u_{t} \in L^{2}\left([0, T], H_{0}^{1}(\Omega)\right)$ and there holds

$$
\begin{aligned}
& \left\langle u_{t t}(t), \eta\right\rangle+\int_{\Omega} \nabla u(t) \nabla \eta d x+\omega \int_{\Omega} \nabla u_{t}(t) \nabla \eta d x+\mu \int_{\Omega} u_{t}(t) \eta d x \\
= & \int_{\Omega} u(t) \ln |u(t)| \eta d x, \forall \eta \in H_{0}^{1}(\Omega), \text { a.e. } t \in[0, T),
\end{aligned}
$$

where $u(x, 0)=u_{0}(x)$ in $H_{0}^{1}(\Omega), u_{t}(x, 0)=u_{1}(x)$ in $L^{2}(\Omega)$. 


\section{Main results}

In this section, we state our main results about problem (1.1)-(1.3).

Theorem 3.1. Let $u_{0}(x) \in H_{0}^{1}(\Omega), u_{1}(x) \in L^{2}(\Omega)$ and (1.4) holds. Then there exists a time $T>0$ such that problem (1.1)-(1.3) admits a unique weak solution $u$ on $[0, T]$ satifying

$$
u \in C\left([0, T], H_{0}^{1}(\Omega)\right) \cap C^{1}\left([0, T], L^{2}(\Omega)\right) \cap C^{2}\left([0, T], H^{-1}(\Omega)\right) .
$$

Theorem 3.2. Let $u_{0}(x) \in H_{0}^{1}(\Omega), u_{1}(x) \in L^{2}(\Omega)$ and (1.4) holds. Assume that $E(0)<d$, then when $u_{0}(x) \in W$, problem (1.1)-(1.3) admits a global weak solution $u(t) \in L^{\infty}\left(0,+\infty ; H_{0}^{1}(\Omega)\right)$ with $u_{t}(t) \in L^{\infty}\left(0,+\infty ; L^{2}(\Omega)\right)$ for $0 \leq t<\infty$.

Theorem 3.3. Let $u_{0}(x) \in H_{0}^{1}(\Omega), u_{1}(x) \in L^{2}(\Omega)$ and (1.4) holds. Assume that $E(0)<d$, then when $u_{0}(x) \in W$, there exist two positive constants $\hat{C}$ and $\xi$ independent of $t$ such that

$$
0<E(t) \leq \hat{C} e^{-\xi t}, t \geq 0 .
$$

Theorem 3.4. Let $u_{0}(x) \in H_{0}^{1}(\Omega), u_{1}(x) \in L^{2}(\Omega)$ and (1.4) holds. Assume that $E(0)<d$, then when $u_{0}(x) \in V$, the existence time of solution to problem (1.1)-(1.3) is infinite and

$$
\lim _{t \rightarrow+\infty}\|u(t)\|^{2}=+\infty \text {. }
$$

Theorem 3.5. Let $u_{0}(x) \in H_{0}^{1}(\Omega), u_{1}(x) \in L^{2}(\Omega)$ and (1.4) holds. Assume that $E(0)=d$, then when $u_{0}(x) \in W$, problem (1.1)-(1.3) admits a global weak solution $u(t) \in L^{\infty}\left(0,+\infty ; H_{0}^{1}(\Omega)\right)$ with $u_{t} \in L^{\infty}\left(0,+\infty ; L^{2}(\Omega)\right)$ for $0 \leq t<\infty$.

Theorem 3.6. Let $u_{0}(x) \in H_{0}^{1}(\Omega), u_{1}(x) \in L^{2}(\Omega)$ and (1.4) holds. Assume that $E(0)=d$, then when $u_{0}(x) \in W$, there exist two positive constants $\hat{C}$ and $\xi$ independent of $t$ such that

$$
0<E(t) \leq \hat{C}_{\star} e^{-\xi t}, t \geq 0 .
$$

Theorem 3.7. Let $u_{0}(x) \in H_{0}^{1}(\Omega), u_{1}(x) \in L^{2}(\Omega)$ and (1.4) holds. Assume that $E(0)=d$, then when $u_{0}(x) \in V$, the existence time of solution to problem (1.1)-(1.3) is infinite and

$$
\lim _{t \rightarrow+\infty}\|u(t)\|^{2}=+\infty .
$$

Theorem 3.8. Let $u_{0}(x) \in H_{0}^{1}(\Omega), u_{1}(x) \in L^{2}(\Omega)$. Assume that $\omega=0, \mu \geq 0$ and $u_{0}(x), u_{1}(x)$ satisfy that

(I) $\quad:\left\|u_{0}\right\|^{2}>4 E(0)>0$,

(II) $\quad:\left(u_{0}(x), u_{1}(x)\right)>0$,

(III) $\quad: u_{0}(x) \in V$,

then the existence time of solution to problem (1.1)-(1.3) is infinite and

$$
\lim _{t \rightarrow+\infty}\|u(t)\|^{2}=+\infty .
$$

Remark 3.9. When $E(0) \leq d$, we derive the global well-posedness and the decay of solution provided $u_{0}(x) \in$ $W$. However when $E(0)>0$ and $u_{0}(x) \in W$, it is still open that whether global solution exists. 


\section{Local existence}

For a fixed $T>0$, consider the space

$$
\mathcal{H}=C\left([0, T], H_{0}^{1}(\Omega)\right) \cap C^{1}\left([0, T], L^{2}(\Omega)\right),
$$

endowed with the norm

$$
\|u\|_{\mathcal{H}}^{2}=\max _{t \in[0, T]}\left(\|\nabla u(t)\|^{2}+\left\|u_{t}(t)\right\|^{2}\right)
$$

\subsection{Proof of Theorem 3.1.}

To establish the uniqueness and existence of local solution to problem (1.1)-(1.3), we first prove the following lemma.

Lemma 4.1. For any $T>0$ and $u \in \mathcal{H}$, there exists a unique

$$
v \in C\left([0, T], H_{0}^{1}(\Omega)\right) \cap C^{1}\left([0, T], L^{2}(\Omega)\right) \cap C^{2}\left([0, T], H^{-1}(\Omega)\right)
$$

such that $v_{t} \in L^{2}\left([0, T], H_{0}^{1}(\Omega)\right)$ solves the linear problem

$$
\begin{cases}v_{t t}-\Delta v-\omega \Delta v_{t}+\mu v_{t}=u \ln |u|, & x \in \Omega, t \in[0, T), \\ v(x, 0)=u_{0}(x), v_{t}(x, 0)=u_{1}(x), & x \in \Omega, \\ v(x, t)=0, & x \in \partial \Omega, t \in[0, T),\end{cases}
$$

where $u_{0}(x)$ and $u_{1}(x)$ are defined in (1.3).

Proof. (4.3) is an inhomogenous linear problem for $v$, carrying a good energy structure. Therefore it suffices to show that the forcing term is $L^{2}$, so that one can apply a standard Galerkin method to extract a solution. So we estimate $\int_{\Omega}(u \ln |u|)^{2} d x$. First by a direct calculation and sobolev inequality, we have

$$
\begin{aligned}
\int_{\Omega}(u \ln |u|)^{2} d x & =\int_{\{x \in \Omega ;|u(x)| \leq 1\}}(u \ln |u|)^{2} d x+\int_{\{x \in \Omega ;|u(x)|>1\}}(u \ln |u|)^{2} d x \\
& \leq e^{-2}|\Omega|+\left(\frac{n-2}{2}\right)^{2} \int_{\{x \in \Omega ;|u(x)|>1\}} u^{\frac{2 n}{n-2}} d x \\
& \leq e^{-2}|\Omega|+\left(\frac{n-2}{2}\right)^{2} c_{\star}^{\frac{2 n}{n-2}}\|\nabla u\|^{\frac{2 n}{n-2}},
\end{aligned}
$$

where $c_{\star}$ is the best constant of the Sobolev embedding $H_{0}^{1}(\Omega) \hookrightarrow L^{\frac{2 n}{n-2}}(\Omega)$. Next we claim the uniqueness. Arguing by contradiction, we suppose that there exist two solutions $v$ and $w$ such that (4.3) hold. Then by subtracting the obtained two equations and testing with $v_{t}-w_{t}$, we can derive

$$
\|\nabla v(t)-\nabla w(t)\|^{2}+\left\|v_{t}(t)-w_{t}(t)\right\|^{2}+2 \int_{0}^{t}\left\|v_{t}(\tau)-w_{t}(\tau)\right\|_{\star}^{2} d \tau=0,
$$

which directly says $w \equiv v$. So we complete the proof.

Take $\left(u_{0}(x), u_{1}(x)\right)$ satisfying (1.4), set $R^{2}=2\left(\left\|\nabla u_{0}\right\|^{2}+\left\|u_{1}\right\|^{2}\right)$ and consider

$$
\mathcal{U}_{T}=\left\{u \in \mathcal{H}: u(x, 0)=u_{0}(x), u_{t}(x, 0)=u_{1}(x),\|u\|_{\mathcal{H}} \leq R\right\}, \forall T>0 .
$$


Again Lemma 4.1 simplifies that for $\forall u \in \mathcal{U}_{T}$, there exists $v=\Phi(u)$ such that $v$ is the unique solution to problem (4.3). Next we prove that for a suitable $T>0, \Phi$ is a contractive map satisfying $\Phi\left(\mathcal{U}_{T}\right) \subset \mathcal{U}_{T}$.

First we can conclude that

$$
\begin{aligned}
& \|\nabla v(t)\|^{2}+\|\dot{v}(t)\|^{2}+2 \int_{0}^{t}\left\|v_{t}(\tau)\right\|_{\star}^{2} d \tau \\
= & \left\|\nabla u_{0}\right\|^{2}+\left\|u_{1}\right\|^{2}+2 \int_{0}^{t} \int_{\Omega} u(\tau) \ln |u(\tau)| v_{t}(\tau) d x d \tau .
\end{aligned}
$$

where $v=\Phi(u)$ is the corresponding solution, to problem (4.3) for fixed $u \in \mathcal{U}_{T}$.

Similar to the arguments of (4.6), we can derive that

$$
\begin{aligned}
& 2 \int_{0}^{t} \int_{\Omega} u(\tau) \ln |u(\tau)| v_{t}(\tau) d x d \tau \\
\leq & \int_{0}^{t}\left(\|u(\tau) \ln |u(\tau)|\|^{2}+\left\|\dot{v}_{h}(\tau)\right\|^{2}\right) d \tau .
\end{aligned}
$$

Hence, (4.7) becomes

$$
2 \int_{0}^{t} \int_{\Omega} u(\tau) \ln |u(\tau)| v_{t}(\tau) d x d \tau \leq c T R^{\frac{2 n}{(n-2)}}+2 \int_{0}^{t}\left\|\dot{v}_{h}(\tau)\right\|_{\star}^{2} d \tau,
$$

for all $t \in(0, T]$. Combining (4.6) with (4.7) and taking the maximum over [0, $T]$ gives

$$
\|v\|_{\mathcal{H}}^{2} \leq \frac{1}{2} R^{2}+c T R^{\frac{2 n}{(n-2)}} .
$$

Choosing $T$ sufficiently small, we get $\|v\|_{\mathcal{H}} \leq R$, which shows that $\Phi\left(\mathcal{U}_{T}\right) \subset \mathcal{U}_{T}$.

Now taking $w_{1}$ and $w_{2}$ in $\mathcal{U}_{T}$, subtracting the two equations (4.3) for $v_{1}=\Phi\left(w_{1}\right)$ and $v_{2}=\Phi\left(w_{2}\right)$, and setting $v=v_{1}-v_{2}$ we obtain for all $\eta \in H_{0}^{1}(\Omega)$ and a.e. $t \in[0, T]$

$$
\begin{aligned}
& \left\langle v_{t t}(t), \eta\right\rangle+\int_{\Omega} \nabla v(t) \nabla \eta d x+\omega \int_{\Omega} \nabla v_{t}(t) \nabla \eta d x+\mu \int_{\Omega} v_{t}(t) \eta d x \\
= & \int_{\Omega}\left(w_{1}(t) \ln \left|w_{1}(t)\right|-w_{2}(t) \ln \left|w_{2}(t)\right|\right) \eta d x \\
= & \int_{\Omega} \xi(t)\left(w_{1}(t)-w_{2}(t)\right) \eta d x,
\end{aligned}
$$

where

$$
0 \leq \xi(t) \leq\left(\ln \left|w_{1}(t)+w_{2}(t)\right|+1\right)\left(w_{1}(t)+w_{2}(t)\right) \ln \left|w_{1}(t)+w_{2}(t)\right| .
$$

Therefore, taking $\eta=v_{t}$ in (4.10) and arguing as above, gives

$$
\left\|\Phi\left(w_{1}\right)-\Phi\left(w_{2}\right)\right\|_{\mathcal{H}}^{2}=\|v\|_{\mathcal{H}}^{2} \leq c R^{\frac{4 n}{(n-2)}} T\left\|w_{1}-w_{2}\right\|_{\mathcal{H}}^{2} \leq \delta\left\|w_{1}-w_{2}\right\|_{\mathcal{H}}^{2}
$$

for some $\delta<1$ as long as $T$ is sufficiently small. So by the Contraction Mapping Principle, we can conclude that problem (1.1)-(1.3) admits a unique solution.

\section{Sub-critical initial energy}

\subsection{Global existence for $E(0)<d$.}

We first prove the invariant set $W$ under the flow of problem (1.1)-(1.3). 
Lemma 5.1. (Invariant set $W$ when $E(0)<d)$ Let $u_{0}(x) \in H_{0}^{1}(\Omega)$ and $u_{1}(x) \in L^{2}(\Omega)$. If $E(0)<d$ and $u_{0}(x) \in W$, then we have

$$
u(t) \in W
$$

and

$$
\|u\|^{2}<4 d \text { for all } t \in[0, T) .
$$

Proof. Let $u(t)$ be the weak solution of problem (1.1)-(1.3) with $E(0)<d, u_{0}(x) \in W$ and $T$ be the maximum existence time of $u(t)$. From Lemma 2.5 we have

$$
E(t) \leq E(0)<d \text { for all } t \in[0, T),
$$

namely,

$$
\frac{1}{2}\left\|u_{t}(t)\right\|^{2}+J(u(t))=E(t)<d \forall t \in[0, T) .
$$

Arguing by contradiction, we suppose that there exists the first $t_{0} \in(0, T)$ such that $I\left(u\left(t_{0}\right)\right)=0$ and $I(u(t))>$ 0 for $0 \leq t<t_{0}$, i.e.,

$$
\left\|\nabla u\left(t_{0}\right)\right\|^{2}=\int_{\Omega} u\left(t_{0}\right)^{2} \ln \left|u\left(t_{0}\right)\right| d x .
$$

From the definition of $\mathcal{N}$, then we have $u\left(t_{0}\right) \in \mathcal{N}$, which implies that $J\left(u\left(t_{0}\right)\right) \geq d$. From (2.8) and the definition of $E(t)$, it holds that

$$
E\left(t_{0}\right)=\frac{1}{2}\left\|u_{t}\left(t_{0}\right)\right\|^{2}+J\left(u\left(t_{0}\right)\right) \geq d,
$$

which contradicts (5.3). Then $u(t) \in W$ for all $t \in[0, T$ ), which together with (5.4) and (2.17) gives

$$
\begin{aligned}
d>E(t) & =\frac{1}{2}\left\|u_{t}(t)\right\|^{2}+J(u) \\
& =\frac{1}{2}\left\|u_{t}(t)\right\|^{2}+\frac{1}{2} I(u)+\frac{1}{4}\|u\|^{2} \\
& \geq \frac{1}{4}\|u\|^{2} .
\end{aligned}
$$

Thus, the proof of Lemma 5.1 is completed.

Now let us turn to prove the existence of global solution to problem (1.1)-(1.3) with $E(0)<d$.

\section{Proof of Theorem 3.2}

From Lemma 5.1 it implies $u(t) \in W$ for any $t \in[0, T)$. By (2.18), we get

$$
\frac{1}{2}\left\|u_{t}\right\|^{2}+\frac{1}{2}\|\nabla u\|^{2}+\frac{1}{4}\|u\|^{2} \leq E(0)+\int_{\Omega} u^{2} \ln |u| d x,
$$

which together with the Logarithmic Sobolev inequality leads to

$$
\left\|u_{t}\right\|^{2}+\left(1-\frac{a^{2}}{2 \pi}\right)\|\nabla u\|^{2}+\frac{n(1+\ln a)+1}{2}\|u\|^{2} \leq C_{1}+\|u\|^{2} \ln \|u\| .
$$

Here and in the sequel we denote by $C_{i}>0, i=1,2,3$ as constants. Choosing $0<a<\sqrt{2 \pi}$ that

we get

$$
1-\frac{a^{2}}{2 \pi}>0
$$

$$
\left\|u_{t}\right\|^{2}+\|\nabla u\|^{2}+\|u\|^{2} \leq C_{2}\left(1+\|u\|^{2} \ln \|u\|^{2}\right) .
$$

Recalling Lemma 5.1, we have

$$
\|u\|^{2}<4 d .
$$

Hence, from inequality (5.8) it follows that

$$
\left\|u_{t}\right\|^{2}+\|\nabla u\|^{2}+\|u\|^{2} \leq C_{3},
$$

where $C_{3}>0$ is a constant independent of $t$. Therefore similar as the proof of Lemma 4.1 we know that problem (1.1)-(1.3) admits a global weak solution. 


\subsection{Asymptotic behavior for $E(0)<d$.}

Next, we can now prove the asymptotic behavior of the solution to problem (1.1)-(1.3), which relies on the construction of a Lyapunov functional by performing a suitable modification of the energy.

Proof of Theorem 3.3

First, we define

$$
L(t):=E(t)+\varepsilon \int_{\Omega} u_{t} u d x+\frac{\varepsilon \omega}{2}\|\nabla u\|^{2},
$$

where $\varepsilon>0$ will be chosen later. From Lemma 5.1 and (2.1)-(2.3) it implies that

$$
0<E(t), \forall t \geq 0 \text {. }
$$

It is easy to see that $L(t)$ and $E(t)$ are equivalent in the sense that there exist two positive constants $\beta_{1}>0$ and $\beta_{2}>0$ depending on $\varepsilon$ such that

$$
\beta_{1} E(t) \leq L(t) \leq \beta_{2} E(t) \text { for } t \geq 0 .
$$

Taking the derivative of $L(t)$ with respect to time yields

$$
\begin{aligned}
\frac{d L(t)}{d t}= & -\omega\left\|\nabla u_{t}\right\|^{2}-\mu\left\|u_{t}\right\|^{2}+\varepsilon\left\|u_{t}\right\|^{2}-\varepsilon\|\nabla u\|^{2} \\
& +\varepsilon \int_{\Omega} u^{2} \ln |u| d x-\varepsilon \mu \int_{\Omega} u_{t} u d x,
\end{aligned}
$$

together with Eq.(1.1). Now, we estimate the last term on the right hand side of (5.12) as follows. By using Young's inequality, we obtain,

$$
\int_{\Omega} u_{t} u d x \leq \frac{1}{4 \delta}\left\|u_{t}\right\|^{2}+\delta\|u\|^{2}, \delta>0 .
$$

Substituting (5.13) into (5.12) with (2.1) gives that

$$
\begin{aligned}
\frac{d L(t)}{d t} \leq & -M \varepsilon E(t)-\omega\left\|\nabla u_{t}\right\|^{2}+\left(\frac{M \varepsilon}{2}+\varepsilon-\mu+\frac{\varepsilon \mu}{4 \delta}\right)\left\|u_{t}\right\|^{2} \\
& +\left(\frac{M \varepsilon}{4}+\varepsilon \mu \delta\right)\|u\|^{2}+\varepsilon\left(\frac{M}{2}-1\right)\|\nabla u\|^{2} \\
& +\varepsilon\left(1-\frac{M}{2}\right) \int_{\Omega} u^{2} \ln |u| d x, \forall M>0 .
\end{aligned}
$$

By logarithmic Sobolev inequality, we have

$$
\begin{aligned}
\frac{d L(t)}{d t} \leq & -M \varepsilon E(t)-\omega\left\|\nabla u_{t}\right\|^{2}+\left(\frac{M \varepsilon}{2}+\varepsilon-\mu+\frac{\varepsilon \mu}{4 \delta}\right)\left\|u_{t}\right\|^{2} \\
& +\left(\frac{M \varepsilon}{4}+\varepsilon \mu \delta+\varepsilon\left(1-\frac{M}{2}\right)\left(\ln \|u\|-\frac{n}{2}(1+\ln a)\right)\right)\|u\|^{2} \\
& +\varepsilon\left(1-\frac{M}{2}\right)\left(\frac{a^{2}}{2 \pi}-1\right)\|\nabla u\|^{2} .
\end{aligned}
$$

Recalling (2.1), (2.4) and $E(t) \leq E(0)<d$, we get

$$
\ln \|u\|^{2}<\ln (4 J(u))<\ln 4 d .
$$

Now, choosing $M<2$, and $\varepsilon$ small enough such that

$$
\left(\frac{M \varepsilon}{2}+\varepsilon-\mu+\frac{\varepsilon \mu}{4 \delta}\right)<0
$$


Then inequality (5.15) becomes

$$
\begin{aligned}
\frac{d L(t)}{d t} \leq & \left(\frac{M \varepsilon}{4}+\varepsilon \mu \delta+\frac{\varepsilon}{2}\left(1-\frac{M}{2}\right)(\ln (4 d)-n(1+\ln a))\right)\|u\|^{2} \\
& -M \varepsilon E(t)+\varepsilon\left(1-\frac{M}{2}\right)\left(\frac{a^{2}}{2 \pi}-1\right)\|\nabla u\|^{2} .
\end{aligned}
$$

Since $0<M<2$ and $J(u) \leq E(0)<d$, by taking

$$
e^{\frac{2 \alpha}{n}} \leq a^{2} \leq 2 \pi
$$

where $\alpha=\ln (4 d)+\frac{M+\mu \delta}{2-M}-n$, and taking $\delta>0$ small enough such that

$$
\frac{a^{2}}{2 \pi}-1 \leq 0
$$

and

$$
\frac{M \varepsilon}{4}+\varepsilon \mu \delta+\frac{\varepsilon}{2}\left(1-\frac{M}{2}\right)(\ln (4 d)-n(1+\ln a)) \leq 0
$$

then we have

$$
\frac{d L(t)}{d t} \leq-M \varepsilon E(t), \forall t>0
$$

Further, by virtue of (5.11), let $\xi=\frac{M \varepsilon}{\beta_{2}}$, (5.20) becomes

$$
\frac{d L(t)}{d t} \leq-\xi L(t), \forall t>0
$$

namely,

$$
L(t) \leq C e^{-\xi t}, \forall t>0
$$

which together with (5.11) shows

$$
0<E(t) \leq \widehat{C} e^{-\xi t}, \forall t>0 .
$$

This completes the proof.

\subsection{Blow-up for $E(0)<d$.}

To prove the blow-up results for problem (1.1)-(1.3) with $E(0)<d$, we first give the invariant set $V$. Similar to Lemma 5.1, we also have the following lemma.

Lemma 5.2. (Invariant set $V$ when $E(0)<d)$ Let $u_{0}(x) \in H_{0}^{1}(\Omega)$ and $u_{1}(x) \in L^{2}(\Omega)$. If $E(0)<d$ and $u_{0}(x) \in V$, then we have

$$
u(t) \in V
$$

and

$$
\|u\|^{2}>4 d \text { for all } t \in[0, T)
$$

Now we turn to show that the blow up in infinite time for problem (1.1)-(1.3) with $E(0)<d$. 


\section{Proof of Theorem 3.4}

Let $u(x, t)$ be a weak solution of problem (1.1)-(1.3) with $E(0)<d, I\left(u_{0}\right)<0$. Arguing by contradiction, we suppose that the solution $u$ is global. Then for any $T>0$ we may consider the auxiliary function $G:[0, T] \rightarrow$ $\mathbb{R}_{+}$defined by

$$
G(t):=\|u\|^{2}+\int_{0}^{t}\|u(\tau)\|_{\star}^{2} d \tau+(T-t)\left\|u_{0}\right\|_{\star}^{2} .
$$

Since $G(t)>0$ is positive over $[0, T]$ and continuous, there exists a $\rho>0$ (independent of the choice of $T$ ) such that

$$
G(t) \geq \rho \text { for all } t \in[0, T] .
$$

Furthermore,

$$
\begin{aligned}
G^{\prime}(t) & =2 \int_{\Omega} u u_{t} d x+\|u\|_{\star}^{2}-\left\|u_{0}\right\|_{\star}^{2} \\
& =2 \int_{\Omega} u u_{t} d x+2 \int_{0}^{t}\left(u(\tau) u_{t}(\tau)\right)_{\star} d \tau
\end{aligned}
$$

and

$$
G^{\prime \prime}(t)=2\left\langle u_{t t}, u\right\rangle+2\left\|u_{t}\right\|^{2}+2\left(u, u_{t}\right)_{\star} \text { for a.e. } t \in[0, T] .
$$

Testing Eq. (1.1) with $u$ and plugging the result into (5.29) we obtain

$$
G^{\prime \prime}(t)=2\left(\left\|u_{t}\right\|^{2}-\|\nabla u\|^{2}+\int_{\Omega} u^{2} \ln |u| d x\right) \text { for a.e. } t \in[0, T] .
$$

Therefore, we get

$$
\begin{aligned}
& G(t) G^{\prime \prime}(t)-\left(G^{\prime}(t)\right)^{2} \\
= & 2 G(t)\left(\left\|u_{t}\right\|^{2}-\|\nabla u\|^{2}+\int_{\Omega} u^{2} \ln |u| d x\right) \\
& +4\left(\eta(t)-\left(G(t)-(T-t)\left\|u_{0}\right\|_{\star}^{2}\right)\left(\left\|u_{t}\right\|^{2}+\int_{0}^{t}\left\|u_{t}(\tau)\right\|_{\star}^{2} d \tau\right)\right),
\end{aligned}
$$

where

$$
\begin{aligned}
\eta(t)= & \left(\|u\|^{2}+\int_{0}^{t}\|u(\tau)\|_{\star}^{2} d \tau\right)\left(\left\|u_{t}\right\|^{2}+\int_{0}^{t}\left\|u_{t}(\tau)\right\|_{\star}^{2} d \tau\right) \\
& -\left(\int_{\Omega} u u_{t} d x+\int_{0}^{t}\left(u(\tau), u_{t}(\tau)\right)_{\star} d \tau\right)^{2} .
\end{aligned}
$$

By Schwarz inequality, we can get

$$
\|u\|^{2}\left\|u_{t}\right\|^{2} \geq\left(\int_{\Omega} u u_{t} d x\right)^{2}
$$




$$
\int_{0}^{t}\|u(\tau)\|_{\star}^{2} d \tau \int_{0}^{t}\left\|u_{t}(\tau)\right\|_{\star}^{2} d \tau \geq\left(\int_{0}^{t}\left(u(\tau), u_{t}(\tau)\right)_{\star} d \tau\right)^{2}
$$

and

$$
\begin{aligned}
& \int_{\Omega} u u_{t} d x \int_{0}^{t}\left(u(\tau), u_{t}(\tau)\right)_{\star} d \tau \\
& \leq\|u\|\left(\int_{0}^{t}\|u(\tau)\|_{\star}^{2} d \tau\right)^{\frac{1}{2}}\left\|u_{t}\right\|\left(\int_{0}^{t}\|u(\tau)\|_{\star}^{2} d \tau\right)^{\frac{1}{2}} .
\end{aligned}
$$

These three inequalities entail $\eta(t) \geq 0$ for any $t \in[0, T]$. As a consequence, we reach the following differential inequality

$$
G(t) G^{\prime \prime}(t)-\left(G^{\prime}(t)\right)^{2} \geq G(t) \xi(t) \text { for a.e. } t \in[0, T],
$$

where $\xi:[0, T] \rightarrow \mathbb{R}_{+}$is the map defined by

$$
\xi(t):=-2\left\|u_{t}\right\|^{2}-2 I(u)-4 \int_{0}^{t}\left\|u_{t}(\tau)\right\|_{\star}^{2} d \tau .
$$

From Lemma 2.5, Lemma 5.2, (2.4) and $E(0)<d$, we know

$$
\begin{aligned}
\xi(t) & =4 J(u(t))-4 E(t)-2 I(u(t))-4 \int_{0}^{t}\left\|u_{t}(\tau)\right\|_{\star}^{2} d \tau \\
& =\|u\|^{2}-4 E(t)-4 \int_{0}^{t}\left\|u_{t}(\tau)\right\|_{\star}^{2} d \tau \\
& \geq\|u\|^{2}-4 E(0) \\
& >\|u\|^{2}-4 d>0 .
\end{aligned}
$$

Since $E(0)<d$, there exists $\delta>0$ (independent of $T$ ) such that

$$
\xi(t) \geq \delta \text { for all } t \geq 0 \text {. }
$$

Hence, by (5.27) and (5.34), it follows that

$$
G(t) G^{\prime \prime}(t)-\left(G^{\prime}(t)\right)^{2} \geq \rho \delta>0 \text { for a.e. } t \in[0, T]
$$

which means $G(t) G^{\prime \prime}(t)-\left(G^{\prime}(t)\right)^{2}>0$. On the other hand, by directly calculation, we can see that

$$
(\ln G(t))^{\prime}=\frac{G^{\prime}(t)}{G(t)}
$$

and

$$
(\ln G(t))^{\prime \prime}=\frac{G(t) G^{\prime \prime}(t)-\left(G^{\prime}(t)\right)^{2}}{G^{2}(t)}>0 .
$$

By (5.36), we know that $(\ln G(t))^{\prime}$ is increasing with respect to $t$, using this fact, integrating (5.35) from $t_{0}$ to $t$, we have

$$
(\ln G(t))^{\prime}=\left(\ln G\left(t_{0}\right)\right)^{\prime}+\int_{t_{0}}^{t} \frac{G(t) G^{\prime \prime}(t)-\left(G^{\prime}(t)\right)^{2}}{G^{2}(t)} d \tau
$$


and

$$
\begin{aligned}
& \ln |G(t)|-\ln \left|G\left(t_{0}\right)\right| \\
= & \int_{t_{0}}^{t}(\ln G(\tau))^{\prime} d \tau \\
= & \int_{t_{0}}^{t} \frac{G^{\prime}}{G(\tau)} d \tau \\
\geq & \frac{G^{\prime}\left(t_{0}\right)}{G\left(t_{0}\right)}\left(t-t_{0}\right)
\end{aligned}
$$

where $0 \leq t_{0} \leq t$. Then

$$
G(t) \geq G\left(t_{0}\right) \exp \left(\frac{G^{\prime}\left(t_{0}\right)}{G\left(t_{0}\right)}\left(t-t_{0}\right)\right)
$$

Since $G(0)=0$ and $G^{\prime}(0)>0$, we can take $t_{0}$ sufficiently small such that $G^{\prime}\left(t_{0}\right)>0$ and $G\left(t_{0}\right)>0$. Then for sufficiently large $t$,

$$
\|u\|^{2}=G^{\prime}(t) \geq \frac{G^{\prime}(t)}{G\left(t_{0}\right)} G(t) \geq G^{\prime}(t) \exp \left(\frac{G^{\prime}(t)}{G\left(t_{0}\right)}\left(t-t_{0}\right)\right) \geq C e^{2 t}
$$

i.e.,

$$
\lim _{t \rightarrow+\infty} G(t)=+\infty .
$$

This shows that the weak solution $u$ to problem (1.1)-(1.3) blows up at $+\infty$.

\section{Critical initial energy}

Lemma 6.1. If $E(0)=d$, then there exists a $t_{0} \in(0, T)$ such that

$$
\int_{0}^{t_{0}}\left\|u_{\tau}\right\|_{\star}^{2} d \tau>0
$$

Proof. Arguing by contradiction we suppose that $\int_{0}^{t}\left\|u_{\tau}\right\|_{\star}^{2} d \tau \equiv 0$ for $0 \leq t<T$, which gives $\left\|u_{t}\right\| \equiv 0$ for $0 \leq t<T$. So we can get $u(t) \equiv u_{0}(x)$, which contradicts the assumption of Lemma 6.1.

\subsection{Global existence for critical initial energy.}

Lemma 6.2. Let $u_{0}(x) \in H_{0}^{1}(\Omega)$ and $u_{1}(x) \in L^{2}(\Omega)$. If $E(0)=d$ and $u_{0}(x) \in W$, then we have

$$
u(t) \in W .
$$

Proof. We prove that $u(t) \in W$ for $0 \leq t<T$. Arguing by contradiction we suppose that there exists a $t_{0} \in$ $(0, T)$ such that $I\left(u\left(t_{0}\right)\right)=0$ and $\left\|u\left(t_{0}\right)\right\| \neq 0$, which says $J\left(u\left(t_{0}\right) \geq d\right.$. Hence, by

$$
\frac{1}{2}\left\|u_{t}\left(t_{0}\right)\right\|^{2}+J\left(u\left(t_{0}\right)\right)+\int_{0}^{t_{0}}\left\|u_{\tau}\right\|_{\star}^{2} d \tau=E(0)=d,
$$

we get $\int_{0}^{t_{0}}\left\|u_{\tau}\right\|_{*}^{2} d \tau=0$ and $\left\|u_{t}\right\|^{2}=0$ for $0 \leq t \leq t_{0}$, which implies $\frac{d u}{d t}=0$ and $u(x, t)=u_{0}(x)$ for $x \in \Omega, 0 \leq$ $t \leq t_{0}$. Thus we can conclude $I\left(u\left(t_{0}\right)\right)=I\left(u_{0}\right)>0$, which contradicts $I\left(u\left(t_{0}\right)\right)=0$. 
Now we turn to prove the existence of global solution to problem (1.1)-(1.3) with $E(0)=d$.

Proof of Theorem 3.5

First Theorem 3.1 gives the existence of the local solution. From Lemma 6.1 we can get (6.1). By (2.18) and $E(0)=d$ we obtain

$$
E\left(t_{0}\right)=d-\int_{0}^{t}\left\|u_{\tau}\right\|_{\star}^{2} d \tau<d .
$$

Moreover, from Lemma 6.2 it follows $u\left(t_{0}\right) \in W$. Let $v(t)=u\left(t+t_{0}\right)$ and $t \geq 0$, then $v(t)$ is a solution of problem (1.1)-(1.3), which combining Theorem 3.2 says that $T=+\infty$.

\subsection{Asymptotic behavior for $E(0)=d$.}

Next, we can now prove the asymptotic behavior of the solution to problem (1.1)-(1.3) with $E(0)=d$.

\section{Proof of Theorem 3.6}

From Theorem 3.5 it implies that there exists a $t_{0}>0$ such that $E\left(t_{0}\right)<d, I\left(u\left(t_{0}\right)\right)>0$ or $\left\|u\left(t_{0}\right)\right\|=0$, which together with Theorem 3.3 says,

$$
0<E(t) \leq \hat{C} e^{-\xi\left(t-t_{0}\right)}, t \geq t_{0}
$$

and

$$
0<E(t) \leq \hat{C}_{\star} e^{-\xi t}, t \geq t_{0},
$$

where $\hat{C}_{\star}=\hat{C} e^{-\xi t_{0}}$.

\subsection{Infinite time blow up for the critical initial energy.}

Lemma 6.3. Let $u_{0}(x) \in H_{0}^{1}(\Omega)$ and $u_{1}(x) \in L^{2}(\Omega)$. If $E(0)=d$ and $u_{0}(x) \in V$, then we have

$$
u(t) \in V .
$$

Proof. Arguing by contradiction, we suppose that there exists a $t_{0} \in(0, T)$ such that

$$
I(u)<0,0<t<t_{0}
$$

and

$$
I\left(u\left(t_{0}\right)\right)=0,
$$

which together with (2.8) gives $J\left(u\left(t_{0}\right)\right) \geq d$. The remainder of this proof is similar to the proof of Lemma 6.2.

Now we turn to show that the blow up in infinite time of the solution to problem (1.1)-(1.3) with $E(0)=d$.

Proof of Theorem 3.7

First Theorem 3.1 gives the existence of the local solution. By the similar arguments as those in the proof of Theorem 3.5, together with Lemma 6.1 and Lemma 6.3. we can set $E\left(t_{0}\right)<d$ and $I\left(u\left(t_{0}\right)\right)<0$. The remainder of the proof is the same as Theorem 3.4.

\section{Infinite time blow up for $E(0)>0(\omega=0)$}

We first prove the following lemmas to obtain that the unstable set $V$ is invariant with respect to $t$ under the flow of problem (1.1)-(1.3) with $E(0)>0, \omega=0$ and $\mu \geq 0$. 
Lemma 7.1. Let $\delta \in \mathbb{R}, T>0$ and let $h$ be a Lipschitzian function over $[0, T)$. Assume that $h(0) \geq 0, h^{\prime}(t)+\delta h(t)>$ 0 a.e. $t \in(0, T)$. Then $h(t)>0, \forall t \in(0, T)$.

Proof. Differentiating $e^{\delta t} h(t)$ gives

$$
\frac{e^{\delta t} h(t)}{d t}=\delta e^{\delta t} h(t)+e^{\delta t} h^{\prime}(t)=e^{\delta t}\left(h^{\prime}(t)+\delta h(t)\right)>0,
$$

combining with $h(0) \geq 0$, we know $e^{\delta t} h(t)>e^{0} h(0) \geq 0$. In other words, $h(t)>0$ for all $t \in(0, T)$.

Lemma 7.2. Let $u_{0}(x) \in H_{0}^{1}(\Omega), u_{1}(x) \in L^{2}(\Omega), \omega=0, \mu \geq 0$ and $\left(u_{0}, u_{1}\right) \geq 0$, then the map $\left\{t \mapsto\|u(t)\|^{2}\right\}$ is strictly increasing as long as $u(t) \in V$, where $u(t)$ is the solution of problem (1.1)-(1.3).

Proof. Let

$$
H(t):=\|u\|^{2},
$$

then we have

$$
H^{\prime}(t)=2\left(u, u_{t}\right)
$$

and

$$
H^{\prime \prime}(t)=2\left\langle u_{t t}, u\right\rangle+2\left\|u_{t}\right\|^{2} .
$$

Notice that, for any $t \in(0, T)$ we have

$$
\left\langle u_{t t}, u\right\rangle=\frac{d}{d t}\left(u, u_{t}\right)-\left\|u_{t}\right\|^{2} .
$$

And multiplying Eq. (1.1) by $u$ yields

$$
\left\langle u_{t t}, u\right\rangle+\|\nabla u\|^{2}+\mu\left(u_{t}, u\right)=(u \ln |u|, u) .
$$

Substituting (7.3) into (7.4) gives

$$
\frac{d}{d t}\left(\left(u, u_{t}\right)+\frac{\mu}{2}\|u\|^{2}\right)=\left\|u_{t}\right\|^{2}-I(u),
$$

which shows

$$
\frac{d^{2}}{d t^{2}}\|u\|^{2}+\mu \frac{d}{d t}\|u\|^{2}=2\left\|u_{t}\right\|^{2}-2 I(u) .
$$

From $u(t) \in V$, (7.6) implies

$$
H^{\prime \prime}(t)+H^{\prime}(t)>0, t \in[0, T) .
$$

Obviously, from Lemma 7.1 and $H^{\prime}(0)=\left(u_{0}(x), u_{1}(x)\right) \geq 0$. We conclude that the map $\left\{t \mapsto\|u(t)\|^{2}\right\}$ is strictly increasing for all $t \in(0, T)$.

In the following, we show the invariance of the unstable set $V$ under the flow of problem (1.1)-(1.3) with $E(0)>$ 0 .

Lemma 7.3. (Invariant set $V$ when $E(0)>0)$ Let $u_{0}(x) \in H_{0}^{1}(\Omega), u_{1}(x) \in L^{2}(\Omega)$ and $u$ be a weak solution of problem (1.1)-(1.3) with the maximum existence time interval $[0, T), T \leq+\infty$. Assume that $E(0)>0, \omega=0$ and the initial data satisfy

$$
\left\|u_{0}\right\|^{2}>4 E(0)
$$

then the solution $u(t)$ of problem (1.1)-(1.3) with $E(0)>0$ belongs to $V$, provided that $u_{0}(x) \in V$. 
Proof. We prove $u(t) \in V$ for $t \in[0, T)$. Arguing by contradiction, we suppose that $t_{0} \in(0, T)$ is the first time such that

$$
I\left(u\left(t_{0}\right)\right)=0
$$

and

$$
I\left(u(t)<0 \text { for } t \in\left[0, t_{0}\right) .\right.
$$

Hence from Lemma 7.2 it follows that the map

$$
\left\{t \mapsto\|u(t)\|^{2}\right\}
$$

is strictly increasing on the interval $\left[0, t_{0}\right)$, which together with (7.2) gives that

$$
H(t)=\|u(t)\|^{2}>\left\|u_{0}\right\|^{2}>4 E(0), \forall t \in\left(0, t_{0}\right) .
$$

Moreover, from the continuity of $u(t)$ in $t$, we obtain

$$
\left\|u\left(t_{0}\right)\right\|^{2}>4 E(0) .
$$

Recalling (2.1) and (2.18), we have

$$
\begin{aligned}
E(0)= & E(t)+\mu \int_{0}^{t}\left\|u_{\tau}\right\|^{2} d \tau \\
= & \frac{1}{2}\left\|u_{t}\right\|^{2}+\frac{1}{2}\|\nabla u\|^{2}-\frac{1}{2} \int_{\Omega} u \ln |u| d x+\frac{1}{4}\|u\|^{2} \\
& +\mu \int_{0}^{t}\left\|u_{\tau}\right\|^{2} d \tau \\
= & \frac{1}{2}\left\|u_{t}\right\|^{2}+\frac{1}{2} I(u)+\frac{1}{4}\|u\|^{2}+\mu \int_{0}^{t}\left\|u_{\tau}\right\|^{2} d \tau,
\end{aligned}
$$

which together with (7.8), $\mu \geq 0$ shows that

$$
\begin{aligned}
E(0) & \geq E\left(t_{0}\right)+\mu \int_{0}^{t_{0}}\left\|u_{\tau}\right\|^{2} d \tau \\
& \geq \frac{1}{2}\left\|u_{t}\left(t_{0}\right)\right\|^{2}+\frac{1}{2} I\left(u\left(t_{0}\right)\right)+\frac{1}{4}\left\|u\left(t_{0}\right)\right\|^{2} \\
& =\frac{1}{2}\left\|u_{t}\left(t_{0}\right)\right\|^{2}+\frac{1}{4}\left\|u\left(t_{0}\right)\right\|^{2} \\
& \geq \frac{1}{4}\left\|u\left(t_{0}\right)\right\|^{2} .
\end{aligned}
$$

Obviously (7.12) contradicts (7.10). So the proof is completed.

In the end we present the infinite time blow up result of the solution to problem (1.1)-(1.3) with $E(0)>0$.

Proof of Theorem 3.8

Recalling the auxiliary function $G(t)$ in (5.26), similarly as the proof of Theorem 3.4, we have

$$
\begin{aligned}
& G^{\prime}(t) G(t)-\left(G^{\prime}(t)\right)^{2} \\
\geq & G(t)\left(G^{\prime \prime}(t)-4\left(\left\|u_{t}\right\|^{2}+\int_{0}^{t}\left\|u_{\tau}(\tau)\right\|_{\star}^{2} d \tau\right)\right) \\
\geq & G(t)\left(-2\left\|u_{t}\right\|^{2}-2 I(u)-4 \int_{0}^{t}\left\|u_{\tau}(\tau)\right\|_{\star}^{2} d \tau\right)
\end{aligned}
$$


and

$$
\xi(t):=-2\left\|u_{t}\right\|^{2}-2 I(u)-4 \int_{0}^{t}\left\|u_{\tau}(\tau)\right\|_{\star}^{2} d \tau .
$$

From (7.11) and (2.1)-(2.3), we can deduce (7.14) to

$$
\xi(t)=\|u\|^{2}-4 E(0) .
$$

At this point, (7.7) and (7.15) indicate that

$$
\xi(t)>\sigma>0
$$

Therefore by (7.13)-(7.16) and (5.27), we have

$$
G(t) G^{\prime \prime}(t)-\left(G^{\prime}(t)\right)^{2}>\rho \sigma>0, t \in[0, T) .
$$

This tells us that

$$
\lim _{t \rightarrow+\infty} G(t)=+\infty .
$$

The remainder of the proof is similar to that of Theorem 3.4.

Acknowledgement: This work was supported by the National Natural Science Foundation of China (11871017), the China Postdoctoral Science Foundation(2013M540270), the Fundamental Research Funds for the Central Universities. And the authors are grateful for the insightful comments and constructive suggestions made by the referee, and also the support and contribution from Dr. Yuxuan Chen and Miss Longqi Shang.

\section{References}

[1] I.R. D’Alembert, Recherches sur la courbe que forme une corde tendue mise en vibrations, Hist. \& Mm. Acad. Roy. Sci. Toulouse. (1747), no. 3, 214-249.

[2] D.H. Sattinger, On global solution of nonlinear hyperbolic equations, Arch. Rational Mech. Anal. 30, (1968), 148-172.

[3] L.E. Payne and D.H. Sattinger, Saddle points and instability of nonlinear hyperbolic equations, Israel J. Math. 22, (1975), 273-303.

[4] H.A. Levine and J. Serrin, Global nonexistence theorems for quasilinear evolution equations with dissipation, Arch. Rational Mech. Anal. 137, (1997), no. 4, 341-361.

[5] P. Pucci and J. Serrin, Global nonexistence for abstract evolution equations with positive initial energy, J. Differential Equations. 150, 1998, 1, 203-214.

[6] E. Vitillaro, Global existence theorems for a class of evolution equations with dissipation, Arch. Rational Mech. Anal. 49, (1999), no. 2, 155-182.

[7] G.F. Webb, Existence and asymptotic behavior for a strongly damped nonlinear wave equation, Canad. J. Math. 23, (1980), no. 3, 631-643.

[8] V. Pata and M. Squassina, On the strongly damped wave equation, Comm. Math. Phys. 253, (2005), no. 3, 511-533.

[9] F. Gazzola and M. Squassina, Global solutions and finite time blow up for damped semilinear wave equations, Ann. Inst. $H$. Poincaré Anal. Non Linéaire 32, (2006), no. 2, 185-207.

[10] J.D. Barrow and P. Parsons, Inflationary models with logarithmic potentials, Physiol Rev. D 52, (1995) no. 10, $5576-5587$.

[11] K. Enqvist and J. McDonald, Q-balls and baryogenesis in the MSSM, Phys. Lett. 425, (1998), no. 3-4, 309-321.

[12] T. Cazenave and A. Haraux, Équations dévolution avec non linéarité logarithmique, Annales de la Faculté des sciences de Toulouse, (1980), no. 1, 21-51.

[13] P. Górka, Logarithmic Klein-Gordon equation, Acta Phys. Pol. 40, (2009), no. 1, 59-71.

[14] T. Hiramatsu, M. Kawasaki, F. Takahashi, Numerical study of Q-ball formation in gravity mediation, J. Cosmol. Astropart. Phys1. 90, (2011), 229-238.

[15] X. Han, Global existence of weak solutions for a logarithmic wave equation arising from Q-ball dynamics, Bull. Korean Math. Soc. 50, (2013), no. 1, 275-283.

[16] H.W. Zhang, G.W. Liu, Q.Y. Hu, Exponential decay of energy for a Logarithmic wave quation, J. Partial Differential Equations. 28, 2015, no. 3, 269-277. 
[17] M.M. Al-Gharabli and S.A. Messaoudi, The existence and the asymptotic behavior of a plate equation with frictional damping and a logarithmic source term, J. Math. Anal. Appl 454, (2017), no. 5, 1-14.

[18] M.M. Al-Gharabli and S.A. Messaoudi, Existence and a general decay result for a plate equation with nonlinear damping and a logarithmic source term, J. Evol. Equ. 454, (2017), no. 4, 1-21.

[19] L. Gross, Logarithmic Sobolev inequalities, Amer. J. Math. 97, (1976), 1061-1083.

[20] E.H. Lieb and M. Loss, Analysis, volume 14 of graduate studies in mathematics, Amer. Math. Soc. Providence, RI, 2001. 